The law of unintended consequences in soccer: impact of three-point-a-win rule on strategies and outcomes

Oleksandr Shepotylo

Kyiv School of Economics and Kyiv Economics Institute 


\title{
The law of unintended consequences in soccer: impact of three-point-a-win rule on strategies and outcomes.
}

\author{
Oleksandr Shepotylo
}

June 24, 2010

Kyiv School of Economics and Kyiv Economics Institute

Vul. Yakira, 13 Office 326

Kiev, Ukraine 04119

oshepotylo@eerc.kiev.ua

\begin{abstract}
This paper analyzes whether the three-point rule in soccer brought more action to the game for a large sample of European championships in 1990-1997, revealing team-specific heterogeneity of responses caused by differences in team tactics in the period prior to the rule change. Teams that relied more heavily on tie-intensive tactics dramatically changed their behavior towards more attacking style in away games, considerably reducing probability of a tie. Interestingly, even though the three-point rule significantly reduced proportion of ties, it did not bring more goals into the game because the increased rewards of scoring when the current score is tied were offset by increased incentives of defending the current score when one team led.
\end{abstract}

JEL codes: C40, L51, L83

Keywords: Heterogeneous response; Soccer; Sports; Three-point system

\section{Introduction}

NAPLES, Fla. (CP) - The NHL's 30 general managers have given a thumbs down to the idea of awarding teams three points for a win. "Because it's a terrible idea," Anaheim general manager Brian Burke said Wednesday as three days of GM meetings wrapped up. "They tried this in British soccer and everything I've heard is that it didn't make a difference."

In the middle of the 90's, The Fédération Internationale de Football Association (FIFA), the world soccer government body, recommended all national soccer federations to reward three points for a win in national tournament games instead 
of two points under the old rule. "Public statements by FIFA officials indicated that...they were increasing the rewards for wins while hoping for more scoring" [Garicano and Palacios-Huerta, 2005]. The push for more scoring aimed at counteracting the long term trend of declining in goals per game that has started after the 1954 World Cup when 5.4 goals per game was scored and hit the rock bottom in the 1990 World Cup in Italy with only 2.2 goals per game ${ }^{1}$, making the game less exciting for fans.

Did the three-point system in soccer make a difference? Was it a terrible idea, as Brian Burke said? The questions have been investigated for Portugal [Guedes and Machado, 2002], Spain [Garicano and Palacios-Huerta, 2005], and Germany [Dilger and Geyer, 2009], with the general conclusion that the threepoint rule has brought two offsetting incentives. On the one hand, when the game is tied, the three-point rule encourages more offensive tactics for both teams. On the other hand, when a team leads, it has incentives to play more defensively than under the old regulations. On aggregate, the three-point rule has very small impact on the number of goals per game.

This paper investigates the impact of the change in the rule of assigning points on game outcomes and the tournament strategies, looking at a larger dataset of games in eight European countries ${ }^{2}$ from 1990 to 1997. It argues that there is no common response of heterogeneous teams to the change in the rule. The effect of the three-point rule considerably varies from one country to another, depending on the proportion of games that ended up as ties in the period prior to introduction of the new rule. Therefore, it is difficult to generalize the impact of the three-point rule, by looking at evidence from just one country. Moreover, it turns out that Portugal, Spain, and Germany were among European championships the least affected by the change.

Moschini [2010], is the only paper the author is aware of that looked at data for 35 countries and found a statistically significant increase in the number of goals and decrease in the percentage of ties caused by the rule change. However, the paper looked at the aggregate data and could not reveal important heterogeneity of responses across teams. The identification strategy in this paper utilizes the cross-country variation in timing of introducing the three-point system, as well as, cross-team variation in the intensity of ties for the period before the change had been introduced. The usage of team-pair fixed effects allows looking at the within team-pair specific effects caused by the rule change, the strategy that is not possible for the aggregate data. The main finding is highly heterogeneous reaction to the new rule in different countries and for different teams, linked to the initial proportion of ties in the national soccer competitions and reliance of teams on tie-intensive tactics prior to the change.

\footnotetext{
${ }^{1}$ In European club competitions, there was a similar trend. Internazionale Milan that dominated European and national competitions in 60's applied catenaccio - an extremely defensive tactics - that helped them winning two European and three national titles. The defensive security is the primary objective of catenaccio. A team that utilizes this strategy would try hard to score in the beginning of the game and then, with a lead of 1-0, would implement catenaccio.

${ }^{2}$ Countires are Germany, France, Italy, Netherlands, Poland, Portugal, Romania, and Spain.
} 
The investigated question is closely related to the literature on heterogeneous responses of economic agents to the changes in policies [Heckman, 2001], sometimes leading to consequences unintended by policymakers. In Melitz [2003], trade liberalization leads to expansion of more productive firms but causes least productive firms to close down. Rajan and Zingales [1998] showed that financial development is the most inductive for growth of more financially dependent industries. In this paper, a similar identification strategy is applied. Since the three-point rule had made a tie less attractive outcome, teams that used more tie-intensive tactics had stronger incentives to switch to more aggressive tactics. After the change in the rule, the game where the away team is in the fourth quartile according to tie-intensities are 11 percent more likely to end up as the home win and 15 percent less likely to end up as the tie.

The rest of the paper is organized as follows. Section 2 gives literature review. Section 3 presents some stylized facts and describe data. Section 4 develops a model and discusses the identification strategy. Section 5 presents results. Section 6 concludes.

\section{Background}

The philosophy of soccer emphasizes the maintenance of balance between offense and defense. The fact that teams score approximately 2.6 goals combined per game makes each goal very valuable and discourages teams from placing too many resources into offensive efforts. At the same time, there has been the overall trend of declining average number of goals per game throughout most of the 20th century from above 4 goals per game to current 2.6 goals per game. The trend is illustrated at Figure 1, which reports average number of goals per game scored in World Cups from 1930 to 2006. The trend become apparent in the 1960's when Italian teams who dominated the international club soccer competition applied catenaccio tactics. Catenaccio is built on protecting one's goal as a priority and attacking the opponent's goal as a secondary objective. The success of catenaccio in Italy and internationally - Italy and Argentina were examples of defensively-minded teams that successfully played in the World Cups - forced other teams to play more defensive soccer as well, reducing the average number of goals between 1960's and 1980's.

A closely related problem was high proportion of ties, especially $0-0$ and 1-1 ties, in national tournaments that became more pronounced in the 1970's. Some National Soccer Federations were even forced to introduced measures, specifically targeting the ties. For instance, in the USSR, the cap on the maximum number of draws per season had been in place from 1978 till 1988; a point for a tie has not been awarderd for teams that exceeded the limit which was initially set as 8 ties per season and later softened to 10 ties per season. The cap had been introduced after the 1977 season when proportion of ties reached 44 percent amidst the accusations of game fixing that involved trading of ties between teams in home and away games.

In the beginning of the 1990's, promoting more aggressive soccer and ob- 
Scoring per game in the World Cups, 1930-2006

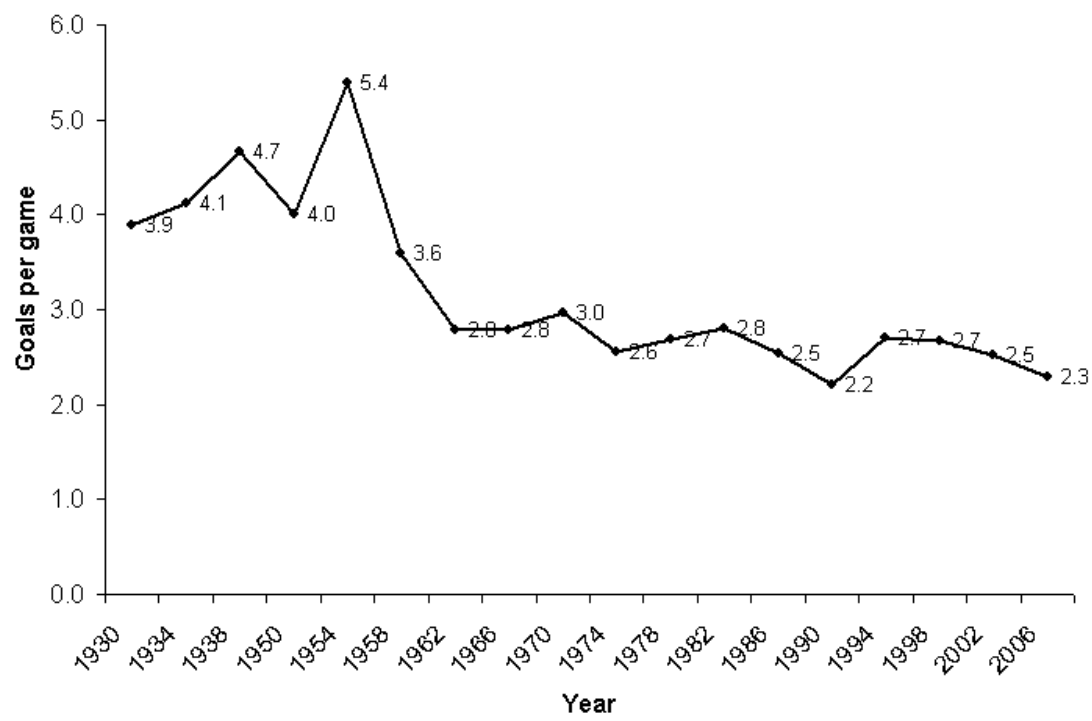

Figure 1: Scoring per game in World Cups, 1930-2006

serving the experience of the English Premiership league that introduced the three-point rule in the 1980's, the European soccer body UEFA recommended awarding three points for a win instead of two points as under the old rule. By 1996, the new system was universally adopted in all countries under the guidance of the international soccer organization, FIFA. While there is some evidence that this change reduced proportion of ties, the effect has had a minimal impact on scoring. To explain the puzzle, Brocas and Carrillo [2004] set up a dynamic game model to study the implications of the three-point rule on the flow of soccer games. They showed that when a team is in the lead the incentive for the team to play offensively may be lower under the three-point rule than under the two-point rule because of the higher expected loss of losing the lead in the game under the new rule.

The empirical estimation of the impact of the three-point rule on games outcomes got attention in the literature [Dilger and Geyer, 2009, Guedes and Machado, 2002, Moschini, 2010, Palacios-Huerta, 2004]. An empirical challenge that researchers are facing is the problem of establishing a causal relationship that goes from changing the rule to changing outcomes of the game. The change in the rule can coincide in time with other important game-related changes such as further commercialization of the game, technological improvements in producing soccer balls ${ }^{3}$ and medical improvements in preparing athletes, changes

\footnotetext{
${ }^{3}$ 'Yet, grab a soccer ball from 1960 , or even one from 1980 or 1990, and the orb is virtually
} 
in tactics and strategy, and changing tastes of fans. Under such circumstances it is quite difficult to disentangle the impact of changing rules from all other factors. Palacios-Huerta [2004] applied a test for structural breaks in time-series to more than a century long series of English soccer data. The results indicated that in 1982 - the year when the English Football Association has introduced the three-point rule - the structural breaks had occurred for percentage of wins and ties, however, there were no breaks in the average number of goals.

More traditional parametric approach found similar evidence of the impact of the three-point rule on game outcomes. Garicano and Palacios-Huerta [2005], who studied Spanish league games, argued that the new rule - introduced to encourage more attacking play and higher number of goals per game - actually produced two effects: more attacking efforts, observed for example in 10 percent increase in shots on goals, as well as more defensive efforts, such as the 12.5 percent increase in the number of fouls per game. ${ }^{4}$ The two effects offset each other and the number of goals per game has not changed due to the new rule. Also, there were no statistically significant changes in the percentage of tied games. To identify the effect, the same teams playing in the National Cup competition were considered as a reference group, because the rule change did not affect the National Cup rules.

Dilger and Geyer [2009] used a similar technique to analyze the German league and confirmed the decrease in the percentage of ties. Guedes and Machado [2002] studied the effects of the new rule on team strategies in the Portuguese first division league. Even though the average number of goals per game increased from 2.40 to 2.60 , or by $8.5 \%$, the favorites scored $13.4 \%$ more goals and losers scored only $1.3 \%$ more. Guedes and Machado [2002] concluded that the new rule has a non-uniform impact on top teams versus lesser teams. Moschini [2010] looked at 30 years of data for 35 countries and found a statistically significant increase in the number of goals and decrease in percentage of ties. However, the main drawback of the paper is the analysis of aggregated final standings rather than microdata of actual games.

\section{Model}

Suppose that a home team $i$ plays against an away team $j . a_{i}$ and $a_{j}$ characterize offensive abilities of the teams, while $d_{i}$ and $d_{j}$ characterize their defensive abilities. The scoring potential of team $i$ in a game against team $j$ defined as

unrecognizable from the one that will be used for the 2010 World Cup in South Africa in June and July. Leather has given way to synthetics. Some 32 individually sewn panels have become eight. Hand stitching has given way to thermal bonding. The result is a faster-than-ever ball for a faster-than-ever game that allows players to attempt laser-like strikes from 40 yards, and precise long-distance passes that would have been impossible with a ball from the 1960s or 1970s." Wall Street Journal, April 21, 2010

4 "soccer clubs reoptimized and changed their behavior in response to stronger incentives, but that they did this largely in a manner undesired by the principal: they engaged in more sabotage activities and managed to decrease the output desired by the principal." [Garicano and Palacios-Huerta, 2005] 
the number of goals scored by the home team, $G_{i j}$, is

$$
G_{i j}=h+g\left(a_{i}, d_{j}\right)+\mu_{i j}
$$

where $h>0$ represents the home field advantage ${ }^{5}$. Function $g(a, d)$ transforms the abilities of the teams into expected number of goals scored by the attacking team, having the following properties $g_{1}>0, g_{11}<0, g_{2}<0$, and $g_{11}<0$. The error term $\mu_{i j}=H_{t}+H_{i j}+\epsilon_{i j}$ consists of the time effect $H_{t}$, pair-specific effect $H_{i j}$, and the random term $\epsilon_{i j} \sim\left(0, \sigma_{\epsilon}^{2}\right)$ is an independent identically distributed shock that includes the impact of such unobservable factors as luck, weather conditions, referee qualification etc.

Analogously, the number of goals scored by the away team, $Q_{i j}$, is

$$
Q_{i j}=g\left(a_{j}, d_{i}\right)+\nu_{i j}
$$

The error term $\nu_{i j}=A_{t}+A_{i j}+\varphi_{i j}$ consists of the time effect $A_{t}$, pair-specific effect $A_{i j}$, and the random term $\nu_{i j} \sim\left(0, \sigma_{\nu}^{2}\right)$ is an independent identically distributed shock.

Suppose further that the function $g$ takes $\log$-linear form $g=\alpha \ln (a)+\beta \ln (d)$. The probability of win for the home team is

$$
P\left(w_{i n}\right)=P\left(G_{i j t}>Q_{i j t}\right)=F_{W}\left(h+\alpha \ln \left(a_{i} / a_{j}\right)+\beta \ln \left(d_{j} / d_{i}\right)+D_{t}+D_{i j}\right)
$$

where $F$ is logistic cdf, $D_{t}=H_{t}-A_{t}$, and $D_{i j}=H_{i j}-A_{i j}$. Similarly, I define equations for ties and losses as

$$
P\left(t i e_{i j t}\right)=P\left(G_{i j t}=Q_{i j t}\right)=F_{T}\left(h+\alpha \ln \left(a_{i} / a_{j}\right)+\beta \ln \left(d_{j} / d_{i}\right)+D_{t}+D_{i j}\right)
$$

and

$$
P\left(\text { loss }_{i j t}\right)=P\left(G_{i j t}<Q_{i j t}\right)=F_{L}\left(h+\alpha \ln \left(a_{i} / a_{j}\right)+\beta \ln \left(d_{j} / d_{i}\right)+D_{t}+D_{i j}\right)
$$

To introduce strategic considerations of teams and test whether they are important parts of team's motivation, we add a strategy related variable $R_{c t}$ that takes value of 1 if the soccer tournament in county $c$ at time $t$ is played under the three-point rule, and takes value of 0 otherwise. The probability of a certain outcome, $O=$ Win, Tie, Loss, is modified as follows

$$
P\left(O_{i j t}\right)=F_{O}\left(\gamma R_{c t}+h+\alpha \ln \left(a_{i} / a_{j}\right)+\beta \ln \left(d_{j} / d_{i}\right)+D_{t}+D_{i j}\right)
$$

To evaluate the impact of the three-point rule on scoring of home and away teams, I estimate the poisson regressions of the following form

$$
G F_{i j t}=\gamma R_{c t}+h+\alpha \ln \left(a_{i} / a_{j}\right)+\beta \ln \left(d_{j} / d_{i}\right)+D_{t}+D_{i j}+\tau_{i j t}
$$

\footnotetext{
${ }^{5}$ The median value of the home field advantage in the sample is 1 , while the average value is 0.62
} 
and

$$
G A_{i j t}=\gamma R_{c t}+h+\alpha \ln \left(a_{i} / a_{j}\right)+\beta \ln \left(d_{j} / d_{i}\right)+D_{t}+D_{i j}+\omega_{i j t}
$$

where $G F_{i j t}$ and $G A_{i j t}$ are goals scored for and against in the game between teams $i$ and $j$ in the season $t$.

Finally, to look if the three-point rule has a heterogeneous effect on different teams, I estimate the following model

$$
\begin{aligned}
P\left(O_{i j t}\right)= & F_{O}\left(\gamma_{0} R_{c t}+\gamma_{1} R_{c t} \times \overline{t i e_{i}}+\gamma_{2} R_{c t} \times \overline{t i e_{i}}+\right. \\
& \left.h+\alpha \ln \left(a_{i} / a_{j}\right)+\beta \ln \left(d_{j} / d_{i}\right)+D_{t}+D_{i j}\right)
\end{aligned}
$$

where $\overline{t i e_{i}}$ and $\overline{t i e_{j}}$ are average proportions of ties of home and away teams in the period before the three-point rule has been introduced and $O=$ Win, Tie, Loss. As an illustration, a negative signs of $\gamma_{1}$ and $\gamma_{2}$ for the probability of a tie would indicate that teams pursuing a strategy of drawing a tie more extensively before the new rule has been introduced, has switched to a more aggresive tactics.

As the main objective, the two following hypotheses are tested.

Hypothesis 1. $H_{0}: \gamma=0$. Teams do not consider tournament strategies and use a "naive" maximum effort tactics.

Hypothesis 2. $H_{0}: \gamma_{1}=0, \gamma_{2}=0$. Teams with higher share of ties in the period prior to the rule change do not respond to the new rule more strongly relative to other teams.

Unconditionally, the new rule discourages ties because it is not optimal to tie in the tournament settings when the team's objective is to win the tournament. However, over the course of the game, the new rule encourages attacking mode only when the current score is tied. When one team leads, the new rule discourages attacking mode for the leading team relative to the two-points per win, while it has only an indirect effect on the incentives for the team that trails behind, stemming from the strategic considerations over the course of a season. As a result, the overall effect on the number of goals scored is not clear. However, the three-point rule does reduce probability of a 0-0 score towards 1-0 and 0-1 outcomes, and reduce probability of a 1-1 score towards 1-2 and 2-1 scores. Also, the three-point rule impact is stronger for teams that were more intensively using defensive tactics trying to draw a tie in the period before the three-point rule has been introduced.

\section{Data and summary statistics}

\subsection{Data source}

The unit of observation is a game between a home team $i$ and away team $j$ in a year $t$. I observe the game outcome - win, tie, or loss from the standpoint of the home team $i$-and number of goals for and against the home team. I also have the final standings of all teams in a season, total number of games per season, 
total number of wins, ties, and losses per season, and total number of goals for and against. The initial sample covers 18,712 games that were played in the top soccer divisions of eight European countries from 1990 to 1997. The time coverage slightly varies across countries - 1990-1997 for France, Germany, Italy, Romania, and Spain, 1990-1996 for Poland, and 1993-1997 for Netherlands and Portugal $^{6}$. Data for Poland are available from http://www.rsssf.com/. For all other countries two sources are used: http://www.sportamok.com for 1990-1992, and http://www.football-data.co.uk/data for 1993-1997.

Table 1 reports the summary statistics before and after the rule has been changed. The first column presents the number of games played during the investigated period in each country. The other columns report proportion of home wins, ties and home losses, average goals scored and conceded by the home team. The proportion of the home wins barely changed increasing from 0.5 to 0.51 . This number is quite stable across countries, except Romania with the proportion of home wins at 0.65 . The proportion of ties dropped from 0.3 under the old rule to 0.26 under the new rule. Importantly, the highest decline in the proportion of ties occurred in France, Italy, and Poland - countries that had a higher than average proportion of ties before the new rule has been introduced. The home losses increased from 0.20 to 0.23 , meaning that most adjustments in the game strategy under the new rule came from a more aggressive game tactics utilized by away teams. The shift in the outcomes from ties to home wins and losses almost did not change the average number of goals per game. The average number of goals scored by the home team increased from 1.58 to 1.63 goals per game, while the average number of goals conceded by the home team increased from 0.95 to 1.03 goals per game.

Statistics of game outcomes reveal high degree of heterogeneity of championships with respect to the initial distribution of wins, ties and losses, and, as I would argue, led to heterogeneous responses in the equilibrium strategies that occurred after introduction of the new rule. In France, Italy, and Poland, the game style was initially skewed towards a defensive strategy, meaning that players assign more attention and effort to destroying the opponent's attempts to score rather than trying to score themselves. This strategy lead to higher share of ties, 0-0 and 1-1 outcomes particularly common, and lower number of goals per game. Since the relative attractiveness of a tie has been reduced under the new rule, French, Italian, and Polish teams had more incentives to adjust their game style away from the strategies that were successful in producing ties and towards a more attacking style, and they actually did adjust considerably. Germany, Netherlands, and Romania, were examples of the other extreme, where most teams prefer attacking style with more goals per game and, as a result, lower number of ties. This group of countries had fewer incentives to change, which showed up in the data. Finally, Spain and Portugal are examples of soccer tournaments with more balanced strategies.

Also, there are important strategic considerations related to the whole tour-

\footnotetext{
${ }^{6}$ Year is given for the beginning of a season. The season starts in summer of one year and ends in spring of the next year. For example, the year 1997 in my sample corresponds to the season $1997 / 1998$
} 
nament strategy that had been changed due to the introduction of the new rule. Under the old system, a French, Italian, or Polish team pursuing a strategy of winning all home games and drawing a tie in all away games would become the champion in half of the cases and come second in the nine out of ten cases, while under the new rule the same tournament strategy would typically bring the third place ${ }^{7}$.

Table 1: Descriptive statistics of game outcomes under two- and three-point systems

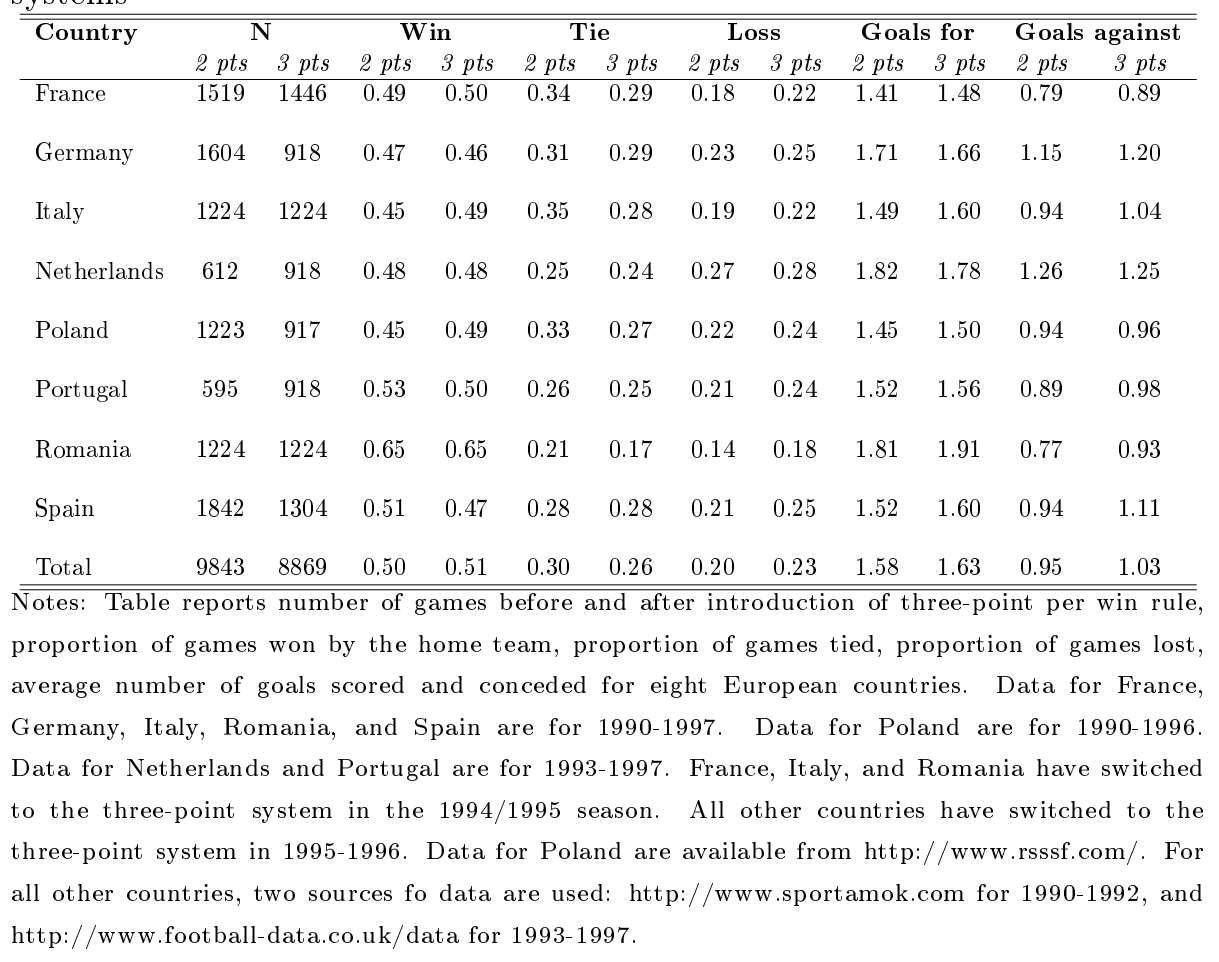

Figure 1 gives an additional insight into the reaction of teams to the new rule by showing the whole distribution of outcomes rather than just sample means. It plots kernel densities of four variables: total points per season, wins per season, draws per season, and losses per season normalized by the total number of games. The sample is split into two parts and densities are constructed separately for the periods before and after the change in the rule took place. For comparability, the total points per season under the three-point-system were converted to the total number of points under the two-point-system. Under the new rule, the variance of the total number of points increased by small amount mostly due to the changes in the upper part of distribution which hints that

\footnotetext{
${ }^{7}$ In the USSR, when 44 percent of all games in the 1977 season ended up as draws, general manager Valeriy Lobanovskij was accused of using such tactics. His team Dynamo Kiev won the tournament with 14 wins and 15 ties, loosing just one game.
} 
the top teams benefited more from the new rule. However, those changes were quite small, indicating that the competitive balance in the European soccer had not changed significantly during the investigated period ${ }^{8}$. The most noticeable change had occurred in the distribution of ties that shifted to the left. The adjustment went through the rightward shift in the distributions of home wins and home losses. More formally, I have tested for the equality of distributions of total number of points, wins, ties, losses, goals for, and goals against before and after the change. A set of the Kolmogorov-Smirnov tests rejects the equality of distributions of wins, ties, and losses, but can not reject the hypothesis of the equality of distributions in the total number of points, as well as, in the number of goals scored for and against the home team.

\subsection{What scores are more popular and how does it change after introducing the three-point system?}

Panel A of Table 2 shows the distribution of game outcomes before the threepoint was introduced calculated as a percentage of the total number of games. The columns of the table are ordered in the number of goals scored by the home team and the rows of the table are ordered in the number of goals scored by the away team. The last column of the table displays the marginal distribution of the goals scored by the home team while the last row of the table shows the marginal distribution of the goals scored by the away team. Before the introduction of the three-point rule, $13.56 \%$ of games ended up as a 1-1 draw, $12.15 \%$ as a $1-0$ home win, and $11.28 \%$ as a $0-0$ draw.

After the introduction of the new rule the rankings of the most popular outcomes has been slightly changed as the panel B of Table 2 demonstrates. It displays the same statistics after the new rule has been introduced for the same group of countries. Under the new rule, $12.15 \%$ of games ended up as a 1-1 draw, $11.72 \%$ as a $1-0$ home win, and $9.45 \%$ as a $2-0$ home win, while a $0-0$ draw took the fourth place with $8.65 \%$ of all games. The panel $\mathrm{C}$ of Table 2 displays the differences between panels $\mathrm{A}$ and $\mathrm{B}$. The three-point rule led to reduction in such popular draw outcomes as $0-0$, and $1-1$ by a total of $4.04 \%$. The adjustment mostly came through the increase in the percentage of away wins $0-1$, and 1-2 by a total of $1.53 \%$. The inspection of the marginal distributions gives as additional insights: an outcome when one of the teams scores three or more goals became more frequent and the effect is more pronounced for away teams.

Initially, I conclude that the introduction of the new rule had the most impact on incentives to score for away teams. When the game starts at $0-0$, both teams have more incentives to score under the new rule, because the benefits of scoring are higher - two extra points to the team's total number of points in the season rather than one extra point under the old rule. However, once a team takes a lead, it has more incentive to play defensively under the new rule, because

\footnotetext{
${ }^{8}$ Higher competitive balance measured as more equal distribution of total points across teams in the tournament would be beneficial for soccer because it makes the oucome of the game more uncertain and more fun to watch, leading to more spectators and as a result to higher revenues from gate receipts and TV commercials.
} 

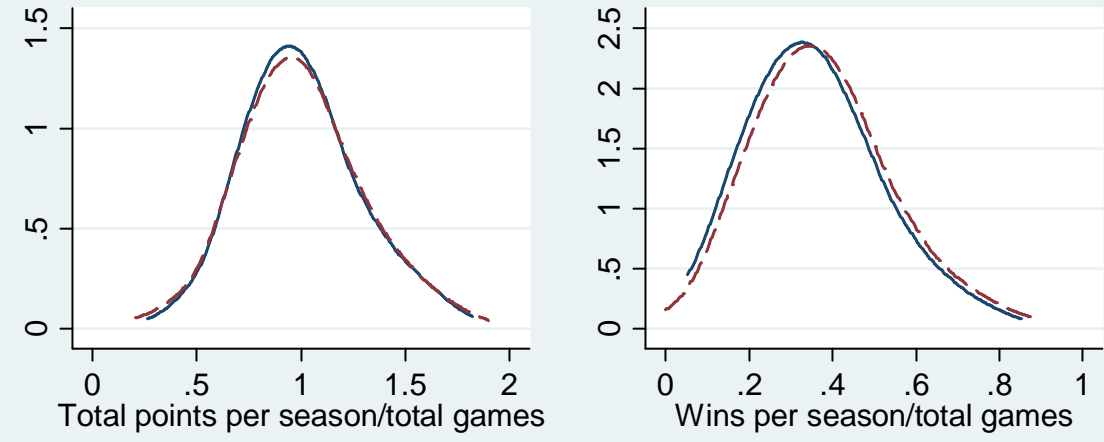

2 point --3 point
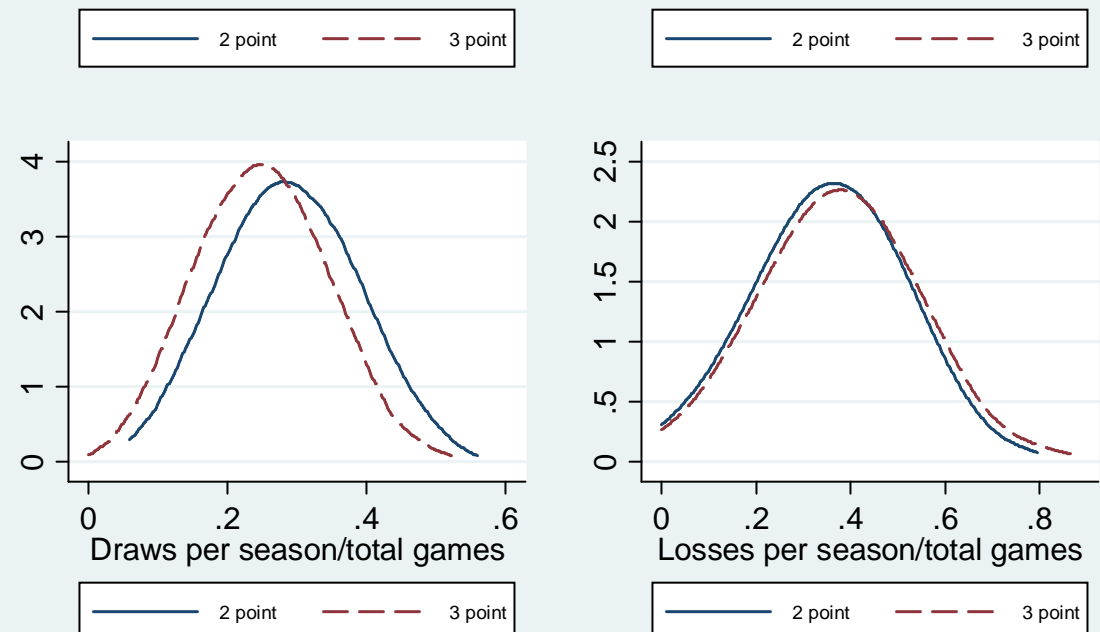

2 point $\quad--3$ point

Notes: Kernel densities of four variables are presented - total points per season, wins per season, draws per season, and losses per season. Values are normalized by the total number of games to account for difference in the number of soccer clubs across countires. The sample is split into two parts and densities are constructed separately for the periods before and after the change in the rule took place. For comparability, the total points per season under the three-point system were converted to the total points per season under the two-point system.

Figure 2: Distributions of game outcomes before and after change in point assigning rule 
Table 2: Game outcomes before and after the change in the rule

\begin{tabular}{lccccccc}
\hline \hline \multicolumn{7}{c}{ A. Game outcomes under two-point rule, \% of total number of games } \\
& \multicolumn{7}{c}{ Goals scored by away team } \\
$\begin{array}{l}\text { Goals scored } \\
\text { by home team }\end{array}$ & 0 & 1 & 2 & 3 & 4 & 5 & Total \\
\hline 0 & 11.28 & 5.55 & 3.27 & 1.53 & 0.67 & 0.12 & 22.4 \\
1 & 12.15 & 13.56 & 4.50 & 1.98 & 0.68 & 0.12 & 33.0 \\
2 & 9.21 & 8.66 & 4.40 & 1.12 & 0.40 & 0.10 & 23.9 \\
3 & 4.90 & 4.74 & 2.20 & 0.72 & 0.15 & 0.01 & 12.7 \\
4 & 2.35 & 2.18 & 0.86 & 0.27 & 0.03 & 0.04 & 5.7 \\
5 & 1.01 & 0.76 & 0.35 & 0.08 & 0.04 & 0.00 & 2.2 \\
\hline Total & 40.9 & 35.5 & 15.6 & 5.7 & 2.0 & 0.4 & 100
\end{tabular}

B. Game outcomes under three-point rule, \% of total number of games

\begin{tabular}{lcccccccc} 
& \multicolumn{7}{c}{ Goals scored by away team } & Total \\
$\begin{array}{l}\text { Goals scored } \\
\text { by home team }\end{array}$ & 0 & 1 & 2 & 3 & 4 & 5 & \\
\hline 0 & 8.65 & 6.45 & 3.52 & 1.65 & 0.63 & 0.27 & 21.2 \\
1 & 11.72 & 12.15 & 5.13 & 2.37 & 0.74 & 0.19 & 32.3 \\
2 & 9.45 & 8.46 & 4.55 & 1.32 & 0.57 & 0.17 & 24.5 \\
3 & 5.17 & 5.07 & 2.49 & 0.71 & 0.25 & 0.08 & 13.8 \\
4 & 2.08 & 2.24 & 0.97 & 0.33 & 0.07 & 0.05 & 5.7 \\
5 & 0.95 & 0.92 & 0.42 & 0.13 & 0.06 & 0.01 & 2.5 \\
\hline Total & 38.0 & 35.3 & 17.1 & 6.5 & 2.3 & 0.8 & 100
\end{tabular}

C. Changes in games outcomes after switch to three-point system

\begin{tabular}{lcccccccc} 
& \multicolumn{7}{c}{ Goals scored by away team } & Total \\
$\begin{array}{l}\text { Goals scored } \\
\text { by home team }\end{array}$ & 0 & 1 & 2 & 3 & 4 & 5 & \\
\hline 0 & -2.63 & 0.90 & 0.25 & 0.12 & -0.04 & 0.15 & -1.24 \\
1 & -0.43 & -1.41 & 0.63 & 0.39 & 0.06 & 0.07 & -0.70 \\
2 & 0.24 & -0.20 & 0.15 & 0.20 & 0.17 & 0.07 & 0.63 \\
3 & 0.27 & 0.33 & 0.29 & -0.01 & 0.10 & 0.07 & 1.06 \\
4 & -0.27 & 0.06 & 0.11 & 0.06 & 0.04 & 0.01 & 0.01 \\
5 & -0.06 & 0.16 & 0.07 & 0.05 & 0.02 & 0.01 & 0.24 \\
\hline Total & -2.88 & -0.16 & 1.51 & 0.80 & 0.35 & 0.38 & 0.00 \\
\hline
\end{tabular}

Notes: Table reports distribution of outcomes of a match between home and away teams as well as distribution of goals scored by one team conditional on the number of goals scored by the other team. They are calculated as a percentage of games with a certain outcome in the total number of games under different point systems. Games where at least one of the teams scored more then 6 goals are not considered. 
it would lose two points if the opponent scores. This line of reasoning is more relevant to model the away team's behavior, because the home team usually chose an attacking mode due to the effect of home team advantage and fan's expectations of watching attacking actions from their team.

\section{$5 \quad$ Results}

For each observation, I observe information on the number of goals scored, $S_{i j t}$, and conceded, $C_{i j t}$, by the home team. I also observe information on the final standings of each team represented by its rank in the end of the season and total number of goals scored, $T S_{i T}$, and conceded, $T C_{i T}$, by the team.

To measure offensive and defensive abilities of teams $i$ and $j$, I construct following measures of offensive and defensive abilities

$$
a_{i t}=\frac{T S_{i T}-S_{i j t}}{T G_{i T}-1}, d_{i t}=\frac{T C_{i T}-C_{i j t}}{T G_{i T}-1}
$$

and

$$
a_{j t}=\frac{T S_{j T}-C_{i j t}}{T G_{i T}-1}, d_{i t}=\frac{T C_{i j T}-S_{i j t}}{T G_{i T}-1}
$$

where $T G_{i T}$ and $T G_{i T}$ are the total number of games played by teams $i$ and $j$ in season $T$. I eliminate the impact of the current game to avoid the problem of endogeneity of the explanatory variables due to the fact that the current result directly enters into calculations of the totals.

Table 3 reports estimation of equations 4, 5, and 6 for the following outcomes - wins, ties, losses, goals scored for and against the home team. Results suggest no significant impact of the new rule on all dependent variables, except the probability of drawing a tie which has reduced by 6 percent after the new rule has been introduced. The relative attack and defense variables are significant and have expected signs.

Introducing interaction of the team-specific proportion of ties for the period before the three-point rule has been introduced with the three-point rule indicator variable reveals that the change in the rule had more pronounced effect for teams that had higher proportion of tied games. Table 4 reports the regression estimates of the effect of the three point rule as well as on the effect of the interaction terms on wins, ties, losses, goals for, and goals against controlling for relative attacking and defensive skills, time effects, and team-pair effects. More tie-intensive teams, i.e. Genoa, Roma, and Torino from Italy and Lille and Martiques from France, are less likely to draw a tie after the three-point rule has been introduced regardless of whether they play at home or away. Interestingly, more tie-intensive teams are more likely to lose the game on the road after the three-point rule has been introduced because more attacking tactics is not their "natural" mode of play. Therefore, the more aggressive tactics selected by tie-intensive away teams makes them more vulnerable to failure.

An approach, alternative to including the interaction terms, is to split the sample according to the tie-intensity of teams and run the same regressions 
Table 3: Impact of the three-point rule on game outcomes

\begin{tabular}{lccccc}
\hline \hline & $(1)$ & $(2)$ & $(3)$ & $(4)$ & $(5)$ \\
& Win & Tie & Loss & Goals for & Goals against \\
\hline \multirow{3}{*}{ Three point $(\mathrm{d})$} & 0.047 & $-0.065^{*}$ & 0.020 & 0.054 & 0.066 \\
& $(0.026)$ & $(0.027)$ & $(0.030)$ & $(0.037)$ & $(0.048)$ \\
Relative attack & $0.14^{* *}$ & $-0.038^{*}$ & $-0.15^{* *}$ & $0.22^{* *}$ & $-0.25^{* *}$ \\
& $(0.018)$ & $(0.019)$ & $(0.021)$ & $(0.026)$ & $(0.034)$ \\
Relative defence & $0.18^{* *}$ & $-0.039^{*}$ & $-0.19^{* *}$ & $0.22^{* *}$ & $-0.22^{* *}$ \\
& $(0.018)$ & $(0.018)$ & $(0.020)$ & $(0.026)$ & $(0.033)$ \\
\hline Log. like & -5428.2 & -4844.3 & -3757.6 & -16806.6 & -13323.3 \\
Observations & 13515 & 12291 & 10323 & 16541 & 16008 \\
\hline \hline
\end{tabular}

Marginal effects; Standard errors in parentheses

(d) for discrete change of dummy variable from 0 to 1

* $p<0.05,{ }^{* *} p<0.01$

Notes: Table reports logit (Columns 1, 2, and 3) and poisson (Columns 4 and 5) regression estimates of the effect of changing the point rule on games outcome - probability of win, tie, and loss, as well as goals scored for and against the home team. All models include time- and team-pair fixed effects. Relative attack and defence variables control for the difference in offensive and defensive abilities of teams during the current season.

on separate samples. This approach allows more flexibility when evaluating heterogeneous responses of teams. I split the sample into four groups according to the quartiles of tie-intensities of away teams because according to the results in Table 4 the away teams are actually more sensitive to the change in the rule. Table 5 shows that after the change in the rule the game where away team is in the fourth quartile according to tie-intensities are 11 percent more likely to end up as the home win and 15 percent less likely to end up as the tie. Again, this result reiterates the previous conclusion that teams that were engaged in tie-intensive strategies under the two point rule were forced to switch to more aggressive game. In addition, they became more vulnerable and suffered higher proportion of defeats.

Finally, proportion of ties before the three point rule has been introduced significantly differed across countries. France, Poland and Italy had the highest proportion of ties of 0.33 and above, while Romania had the lowest proportion of 0.21 . Table 6 reports estimates of the impact of the change in the rule on games outcomes for each country in the sample. As expected, the rule change had significant negative impact on the probability of tie in Poland (by -17 percent), France (by -16 percent), and Italy (by -10 percent). Quite unexpectedly, the probability of tie has decline by 10 percent in the Netherlands as well. In all other countries the effect is insignificant. Another important result is a considerable increase in the probability of loss by the home team in France and Romania (by 21 percent), Italy (18 percent), and Spain (by 11 percent). The increase in the probability of loss in those countries came from more aggressive 
Table 4: Interacting the three-point rule with the team-specific tie intensity

\begin{tabular}{lccccc}
\hline \hline & $(1)$ & $(2)$ & $(3)$ & $(4)$ & $(5)$ \\
& Win & Tie & Loss & Goals for & Goals against \\
\hline \multirow{3}{*}{ Three point $(\mathrm{d})$} & & & & & \\
& $-0.15^{* *}$ & $0.22^{* *}$ & -0.058 & -0.054 & 0.066 \\
Three point x share of ties i & $(0.056)$ & $(0.059)$ & $(0.067)$ & $(0.080)$ & $(0.11)$ \\
& 0.30 & $-0.37^{*}$ & -0.0033 & -0.014 & 0.043 \\
Three point x share of ties j j & $(0.16)$ & $(0.17)$ & $(0.18)$ & $(0.24)$ & $(0.30)$ \\
& $0.41^{*}$ & $-0.60^{* *}$ & 0.22 & 0.41 & -0.10 \\
Relative attack & $(0.16)$ & $(0.17)$ & $(0.19)$ & $(0.23)$ & $(0.31)$ \\
& $0.15^{* *}$ & -0.033 & $-0.16^{* *}$ & $0.23^{* *}$ & $-0.26^{* *}$ \\
Relatve defence & $(0.018)$ & $(0.019)$ & $(0.021)$ & $(0.027)$ & $(0.035)$ \\
& $0.18^{* *}$ & $-0.042^{*}$ & $-0.18^{* *}$ & $0.21^{* *}$ & $-0.22^{* *}$ \\
\hline Log. like & $(0.018)$ & $(0.019)$ & $(0.021)$ & $(0.027)$ & $(0.034)$ \\
Observations & -5129.8 & -4601.5 & -3542.2 & -15855.8 & -12563.5 \\
\hline \hline
\end{tabular}

\footnotetext{
Marginal effects; Standard errors in parentheses

(d) for discrete change of dummy variable from 0 to 1 
Table 5: Quartile regressions

\begin{tabular}{|c|c|c|c|c|c|}
\hline & $\begin{array}{c}(1) \\
\text { 1st Quartile }\end{array}$ & $\begin{array}{c}(2) \\
\text { 2nd Quartile }\end{array}$ & $\begin{array}{c}(3) \\
\text { 3rd Quartile }\end{array}$ & $\begin{array}{c}(4) \\
\text { 4th Quartile }\end{array}$ & \\
\hline Win $(d)$ & $\begin{array}{c}0.064 \\
(0.064)\end{array}$ & $\begin{array}{c}0.046 \\
(0.063)\end{array}$ & $\begin{array}{c}0.032 \\
(0.042)\end{array}$ & $\begin{array}{c}0.11^{*} \\
(0.050)\end{array}$ & \\
\hline Tie (d) & $\begin{array}{c}0.033 \\
(0.074)\end{array}$ & $\begin{array}{c}0.047 \\
(0.073)\end{array}$ & $\begin{array}{l}-0.017 \\
(0.046)\end{array}$ & $\begin{array}{c}-0.15 * * \\
(0.050)\end{array}$ & Notes: \\
\hline $\operatorname{Loss}(d)$ & $\begin{array}{c}-0.15 \\
(0.083)\end{array}$ & $\begin{array}{c}-0.15 \\
(0.083)\end{array}$ & $\begin{array}{l}-0.030 \\
(0.051)\end{array}$ & $\begin{array}{c}0.052 \\
(0.063)\end{array}$ & \\
\hline \multicolumn{6}{|c|}{$\begin{array}{l}\text { Marginal effects; Standard errors in parentheses } \\
\text { (d) for discrete change of dummy variable from } 0 \text { to } 1 \\
* p<0.05, * * p<0.01 \\
\text { Table reports logit regression estimates of the effect of changing the point rule on games outcomes - } \\
\text { probability of win, tie, and loss - estimated separately for four equal groups of games. Observations } \\
\text { are split into four quartiles according to the average proportion of the tied games for the away } \\
\text { team in the period before the three-point rule has been introduced. Each element in the table is } \\
\text { estimated by a separate logit regression and reports the change in the probability of the outcome } \\
\text { due to the change in the point rule. All models include time- and team-pair fixed effects. Relative } \\
\text { attack and defence variables control for the difference in offensive and defensive abilities of teams } \\
\text { during the current season are included but not reported. }\end{array}$} \\
\hline
\end{tabular}


away strategy resulted in higher number of goals scored by the away teams. At the same time, no significant increase in the number of goals scored by the home teams is found in any country in the sample.

\section{Did the three point system influence final scores?}

Table 7 reports the impact of the three-point rule on probability of the final score, which is reported at the top of the table. The effect is estimated on the whole sample, as well as on four sub-samples, selected according to the quartiles of distribution of proportion of ties for an away team computed for the period before the three-point rule has been introduced. Overall, a 10 percent lower probability of a $0-0$ ourcome is statistically different from zero at 5 percent confidence level. However, looking at estimates for four sub-samples gives a different conclusion, the only statistically significant change after the new rule has been introduced occurred for the fourth quartile - probability of a 1-1 outcome declined by 19 percent while probability of a $0-1$ outcome increased by 24 percent. There were no statistically significant shift in the probability of score outcomes for any other sub-sample.

The significant shift in the distribution of final scores from 1-1 to 0-1 can explain why incentives for more attacking tactics that are present when the game begins do not lead to more scoring per game. Under the three-point rule, the away team that scored first, has stronger incentives to defend 0-1 outcome, because it protects two points under the three-point rule relative to only one point under the two-point rule. The change in incentives lead to statistically significant drop in 1-1 outcome and increase in 0-1 outcome.

\section{Conclusions}

This paper demonstrates that lack of an aggregate response to a change in a policy can disguise significant heterogeneity of responses along some dimensions that characterize economic agents, firms, or soccer clubs. The same policy can have counter-balancing effects on two groups of agents with responses that cancel out each other after aggregation. During a soccer game, the three-point rule increases incentives to attack while the game is tied, and increases incentives to protect the current score when the team leads. On average, there is a negligible effect of the three-point rule on number of goals per game. At the same time, the two-point rule skewed incentives of away teams in favor of defending the tie, generating high frequency of outcomes $0-0$ and 1-1. The three-point rule has destroyed those incentives and encouraged away teams to play more aggressively.

In particular, the three-point rule in soccer had a large impact on behavior of teams that, prior to the new rule, relied more heavily on tie-intensive tactics in away games. The change in behavior of this group led to lower proportion of ties, more goals scored by away teams, and significant shift from 1-1 outcome to $0-1$ outcome. Likewise, the national soccer competitions that had higher 
Table 6: Impact of change in the point rule on outcomes by country

\begin{tabular}{|c|c|c|c|c|c|}
\hline & $\begin{array}{c}(1) \\
\text { Win }\end{array}$ & $\begin{array}{l}(2) \\
\text { Tie }\end{array}$ & $\begin{array}{c}(3) \\
\text { Loss }\end{array}$ & $\begin{array}{c}(4) \\
\text { Goals for }\end{array}$ & $\begin{array}{c}(5) \\
\text { Goals against }\end{array}$ \\
\hline France (a) & $\begin{array}{l}-0.017 \\
(0.046)\end{array}$ & $\begin{array}{c}-0.16^{* *} \\
(0.046)\end{array}$ & $\begin{array}{c}0.21^{* *} \\
(0.048)\end{array}$ & $\begin{array}{c}0.027 \\
(0.074)\end{array}$ & $\begin{array}{c}0.20^{*} \\
(0.095)\end{array}$ \\
\hline Log. like & -969.4 & -877.4 & -613.5 & -2739.2 & -2078.0 \\
\hline Observations & 2354 & 2192 & 1711 & 2715 & 2638 \\
\hline Germany & $\begin{array}{l}0.0092 \\
(0.045)\end{array}$ & $\begin{array}{c}-0.0099 \\
(0.045)\end{array}$ & $\begin{array}{c}0.015 \\
(0.053)\end{array}$ & $\begin{array}{c}0.059 \\
(0.072)\end{array}$ & $\begin{array}{c}-0.014 \\
(0.084)\end{array}$ \\
\hline Log. like & -806.7 & -725.8 & -587.1 & -2495.9 & -2085.8 \\
\hline Observations & 1956 & 1822 & 1593 & 2318 & 2288 \\
\hline Italy & -0.038 & $-0.10^{*}$ & $0.18^{* *}$ & 0.064 & $0.41^{* *}$ \\
\hline Log. like & -679.5 & -680.3 & -479.4 & -2067.9 & -1700.2 \\
\hline Observations & 1683 & 1668 & 1340 & 2116 & 2067 \\
\hline Netherlands & $\begin{array}{c}0.082 \\
(0048)\end{array}$ & $\begin{array}{l}-0.10^{*} \\
0.029)\end{array}$ & $\begin{array}{r}0.0018 \\
(0052)\end{array}$ & $\begin{array}{r}-0.070 \\
(0067)\end{array}$ & $\begin{array}{c}0.075 \\
(0080)\end{array}$ \\
\hline Log. like & -397.6 & -355.7 & -338.3 & -1449.7 & -1232.9 \\
\hline Observations & 1050 & 941 & 907 & 1371 & 1366 \\
\hline Poland & 0.075 & $-0.17^{* *}$ & 0.12 & 0.016 & 0.0035 \\
\hline & $(0.055)$ & $(0.049)$ & $(0.064)$ & $(0.081)$ & $(0.11)$ \\
\hline Log. like & -525.5 & -484.0 & -358.3 & -1561.9 & -1228.8 \\
\hline Observations & 1389 & 1263 & 998 & 1749 & 1653 \\
\hline Portugal & -0.022 & 0.0085 & 0.024 & 0.087 & 0.087 \\
\hline Log. like & -319.5 & -282.1 & -228.7 & -1079.3 & -827.3 \\
\hline Observations & 843 & 739 & 625 & 1204 & 1104 \\
\hline Romania & -0.085 & -0.094 & $0.21^{* *}$ & -0.0061 & $0.36^{* *}$ \\
\hline Log. like & $\begin{array}{l}(0.054) \\
-649.5\end{array}$ & $\begin{array}{l}(0.061) \\
-5138\end{array}$ & $\begin{array}{l}(0.053) \\
-383.6\end{array}$ & $\begin{array}{l}(0.070) \\
-2356.1\end{array}$ & $\begin{array}{c}(0.10) \\
-1657.8\end{array}$ \\
\hline Observations & 1711 & 1387 & 1127 & 2218 & 2121 \\
\hline Spain & -0.080 & 0.0021 & $0.11^{*}$ & 0.089 & $0.35^{* *}$ \\
\hline & $(0.042)$ & $(0.047)$ & $(0.048)$ & $(0.069)$ & $(0.085)$ \\
\hline Log. like & -1037.8 & -892.9 & -745.1 & -3027.3 & -2478.2 \\
\hline Observations & 2529 & 2279 & 2022 & 2850 & 2771 \\
\hline
\end{tabular}

Marginal effects; Standard errors in parentheses

(a) for discrete change of dummy variable from 0 to 1

$* p<0.05,{ }^{* *} p<0.01$

Notes: Table reports logit (Columns 1, 2, and 3 ) and poisson (Columns 4 and 5) regression estimates of the effect of changing the point rule on games outcome - probability of win, tie, and loss, as well as goals scored for and against the home team for each country in the sample. All models include time- and team-pair fixed effects. Relative attack and defence variables - not shown in the table - control for the difference in offensive and defensive abilities of teams during the current season. 
Table 7: Impact of three-point rule on final scores

\begin{tabular}{lcccccc}
\hline \hline & & \multicolumn{5}{c}{ Final Score } \\
& $0-0$ & $0-1$ & $1-0$ & $1-1$ & $1-2$ & $2-1$ \\
\hline Whole sample & $-0.10^{*}$ & 0.06 & 0.04 & -0.06 & -0.007 & 0.02 \\
& $(0.04)$ & $(0.05)$ & $(0.04)$ & $(0.04)$ & $(0.06)$ & $(0.04)$ \\
First quartile & -0.03 & -0.15 & -0.05 & -0.03 & -0.10 & 0.10 \\
& $(0.11)$ & $(0.16)$ & $(0.08)$ & $(0.11)$ & $(0.14)$ & $(0.10)$ \\
Second quartile & -0.04 & -0.13 & -0.07 & -0.01 & -0.11 & 0.10 \\
& $(0.11)$ & $(0.16)$ & $(0.08)$ & $(0.11)$ & $(0.14)$ & $(0.09)$ \\
& & & & & & \\
Third quartile & -0.10 & 0.06 & -0.00 & -0.01 & -0.17 & 0.02 \\
& $(0.07)$ & $(0.08)$ & $(0.06)$ & $(0.06)$ & $(0.12)$ & $(0.07)$ \\
& & & & & & \\
Fourth quartile & -0.07 & $0.24^{*}$ & 0.05 & $-0.19^{* *}$ & -0.00 & 0.08 \\
& $(0.07)$ & $(0.10)$ & $(0.07)$ & $(0.07)$ & $(0.11)$ & $(0.09)$ \\
\hline \hline
\end{tabular}

Marginal effects; Standard errors in parentheses

* $p<0.05,{ }^{* *} p<0.01$

Table reports logit regression estimates of the effect of changing the point rule on the probability of a particular final score, shown at the top of the table. Effect is estimated on the whole sample, as well as, on four subsamples split according to quartiles of proportion of ties calculated for away teams for the period before the new rule has been introduced. All regressions include time- and team-pair fixed effects. Relative attack and defence variables - not reported in the table - control for the difference in offensive and defensive abilities of teams during the current season. 
proportion of ties before the three-point rule has been introduced, responded more strongly to the rule change. Interestingly, shifting the away tactics to more aggressive mode increases both the probability of winning and probability of loosing the game.

Heterogeneity of responses is measured on within team-pair variation in teams' behavior, and accounting for time trend, which allows controlling for technological progress, development of better managerial practices, and other factors that can lead to structural changes in game outcomes. In addition, different timing of the rule change in the countries represented in the sample, allows separate the time trend from the effect of the policy change.

\section{References}

I. Brocas and J.D. Carrillo. Do the" Three-Point Victory" and" Golden Goal" Rules Make Soccer More Exciting? Journal of Sports Economics, 5(2):169, 2004.

A. Dilger and H. Geyer. Are Three Points for a Win Really Better Than Two?: A Comparison of German Soccer League and Cup Games. Journal of Sports Economics, 10(3):305, 2009.

L. Garicano and I. Palacios-Huerta. Sabotage in tournaments: Making the beautiful game a bit less beautiful. Discussion paper series, Centre for Economic Policy Research, London, 5231, 2005.

J.C. Guedes and F.S. Machado. Changing rewards in contests: Has the threepoint rule brought more offense to soccer? Empirical Economics, 27(4): 607-630, 2002.

J.J. Heckman. Micro data, heterogeneity, and the evaluation of public policy: Nobel lecture. Journal of Political Economy, 109(4), 2001.

M.J. Melitz. The impact of trade on intra-industry reallocations and aggregate industry productivity. Econometrica, 71(6):1695-1725, 2003.

G. Moschini. Incentives and outcomes in a strategic setting: The 3-points-fora-win system in soccer. Economic Inquiry, 48(1):65-79, 2010.

I. Palacios-Huerta. Structural changes during a century of the world's most popular sport. Statistical Methods and Applications, 13(2):241-258, 2004.

R.G. Rajan and L. Zingales. Financial dependence and growth. American Economic Review, 88(3):559-586, 1998. 